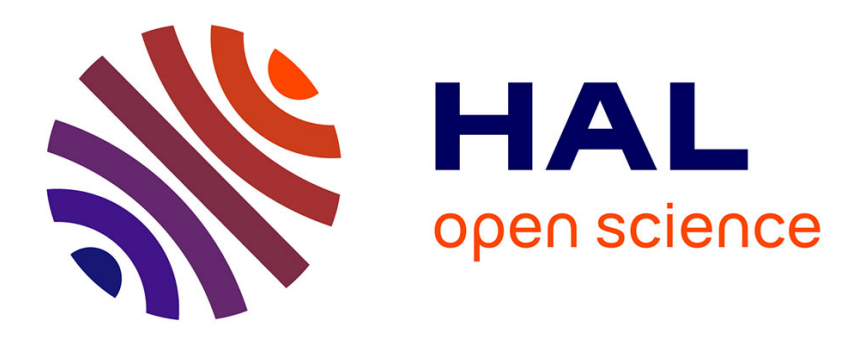

\title{
Increasing peak-field generation efficiency of reverberation chamber
}

\author{
Andrea Cozza
}

\section{To cite this version:}

Andrea Cozza. Increasing peak-field generation efficiency of reverberation chamber. Electronics Letters, 2010, 46 (1), pp.38-39. hal-00505994

\section{HAL Id: hal-00505994 \\ https://hal.science/hal-00505994}

Submitted on 8 Nov 2010

HAL is a multi-disciplinary open access archive for the deposit and dissemination of scientific research documents, whether they are published or not. The documents may come from teaching and research institutions in France or abroad, or from public or private research centers.
L'archive ouverte pluridisciplinaire HAL, est destinée au dépôt et à la diffusion de documents scientifiques de niveau recherche, publiés ou non, émanant des établissements d'enseignement et de recherche français ou étrangers, des laboratoires publics ou privés. 


\title{
Increasing the Peak-Field Generation Efficiency of a Reverberation Chamber
}

\author{
Andrea Cozza
}

The use of time-reversal techniques has been shown to allow focusing energy in a spot about half a wavelength wide. The fact of being able to concentrate energy into a reduced volume of space implies higher power densities and, ultimately, higher field levels. The use of this feature for improving the ability of a reverberation chamber in converting energy into high-intensity fields is investigated here. Experimental results are compared to those predicted by a simple asymptotic model, revealing the role played by losses and frequency bandwidth and how the performance of time-reversal techniques depends on these parameters.

\section{INTRODUCTION}

Among the several advantages presented by reverberation chambers (RCs), a special place is certainly held by their ability to produce high-intensity electromagnetic fields from relatively low-power sources. The standard harmonic excitation of an RC leads to a statistically uniform spreading of the electromagnetic energy over the entire volume of the cavity, essentially because of the fact that the cavity resonances are excited in an incoherent way. This implies that of all the energy stored in an $\mathrm{RC}$ driven by a continuous wave $(\mathrm{CW})$ harmonic signal, only a fraction can be used for the "aggression" of the equipment under test (EUT). But while carrying out Electromagnetic Compatibility tests, as well as other types of radiated tests in RC, it would be typically more useful to be able of concentrating energy only over the EUT. This scenario can be modified thanks to recent advances brought by time-reversal techniques [1]: as a matter of fact, this approach allows to concentrate a bigger share of energy around and towards the EUT, thus increasing the efficiency of the RC as a high-intensity field generator for equipment testing.

In this letter, we prove that higher field-generation efficiencies are indeed made possible by using non-harmonic, time-reversal-based signals; we focus on how the physical parameters 
of the RC (quality factor $Q$, signal bandwidth, etc. ) affect the performance of time-reversal driven RCs. To this end, we propose a simple asymptotic model capable of predicting the average improvement brought by time-reversal techniques over the standard use of RCs. Its validity is checked against experimental results obtained in an actual RC.

\section{PEAK-FIELD GENERATION EFFICIENCY}

For the purpose of our analysis the reverberation chamber will be represented as a blackbox linear system. To this end, the vector electric field $\mathbf{E}(f)$ generated at a certain position, will be described as

$$
\mathbf{E}(f)=X(f) \boldsymbol{\Phi}(f)=X(f)\left(\begin{array}{lll}
\phi_{1} & \phi_{2} & \phi_{3}
\end{array}\right)^{\mathrm{T}}
$$

where $X(f)$ is the power-wave applied at the input port of the antenna exciting the chamber and $\phi_{p}(f)$ is the transfer function related to the $p$-th Cartesian component of the E field. Two cases will be considered for the excitation of the system: 1) $X_{\mathrm{CW}}(f)$, a harmonic steady-state signal of frequency $f_{c}$, with peak amplitude $A$, and 2) $X_{\mathrm{TR}}(f)=P(f) \phi_{p}^{\star}(f)$, with $P(f)$ the spectrum of a pulse signal $p(t)$, covering a bandwidth $B_{T}$ around the central frequency $f_{c}$. This latter case, i.e., of a time-reversal-driven excitation, leads to the generation of a pulse approximating $p(t)$ [2], dominated by the field component along the $p$-th dimension [3]. Defining $p(t)$ as to attain its peak value at $t=0$, the peak field generated by applying $X_{\mathrm{TR}}(f)$ is, in time-domain,

$$
\max _{t}\left\|\mathbf{e}_{\mathrm{TR}}(t)\right\|=\left\|\mathbf{e}_{\mathrm{TR}}(0)\right\|=2 \int_{B_{T}} P(f)\left|H_{p}(f)\right|^{2} \mathrm{~d} f
$$

having carried out the integral over the positive-frequency region of the spectrum. Conversely, $X_{\mathrm{CW}}(f)$ yields a non-polarized field, whose peak value is given by

$$
\max _{t}\left\|\mathbf{e}_{\mathrm{CW}}(t)\right\|=A\left\|\mathbf{\Phi}\left(f_{c}\right)\right\|
$$

Before being compared, the peak fields obtained through these two approaches need to be normalized to the energy $\mathcal{E}$ that is necessary to apply for their generation, thus leading to the definition of the peak-field generation efficiency

$$
\eta=\frac{\max _{t}\|\mathbf{e}(t)\|^{2}}{\mathcal{E}} .
$$

In the case of a harmonic excitation, $95 \%$ of the steady-state amplitude is attained after a period equal to $3 \tau$, with $\tau=Q /\left(\pi f_{c}\right)$ the average time-constant of the $\mathrm{RC}, Q$ being the 
average quality factor of the $\mathrm{RC}$ at $f_{c}$. Hence, an average applied energy

$$
\mathcal{E}_{\mathrm{CW}}=\frac{3}{2} \frac{A^{2} Q}{\pi f_{c}}
$$

which leads to

$$
\eta_{\mathrm{CW}}=\frac{2}{3} \frac{\pi f_{c}}{Q}\left\|\boldsymbol{\Phi}\left(f_{c}\right)\right\|^{2}
$$

Assuming an ideal reverberating chamber [4], the average quadratic field amplitude would be evenly distributed along the three field components, so that introducing the quadratic average $\phi_{\mathrm{av}}^{2}\left(f_{c}\right)$

$$
\mathrm{E}\left[\left|\phi_{p}\left(f_{c}\right)\right|^{2}\right]=\phi_{\mathrm{av}}^{2}\left(f_{c}\right) \quad \forall p \in[1,3]
$$

yields

$$
\mathrm{E}\left[\eta_{\mathrm{CW}}\right]=\frac{2 \pi f_{c}}{Q} \phi_{\mathrm{av}}^{2}\left(f_{c}\right)
$$

Conversely, time-reversal deals with a finite-energy excitation

$$
\mathcal{E}_{\mathrm{TR}}=2 \int_{B_{T}}\left|X_{\mathrm{TR}}(f)\right|^{2} \mathrm{~d} f=2 \int_{B_{T}}|P(f)|^{2}\left|\phi_{p}(f)\right|^{2} \mathrm{~d} f \quad,
$$

so that its efficiency is given by

$$
\eta_{\mathrm{TR}}=2 \frac{\left(\int_{B_{T}} P(f)\left|\phi_{p}(f)\right|^{2} \mathrm{~d} f\right)^{2}}{\int_{B_{T}}|P(f)|^{2}\left|\phi_{p}(f)\right|^{2} \mathrm{~d} f} .
$$

Definitions (6) and (10) allow assessing how these two ways of using RCs manage to convert the same amount of energy into a peak field, just by knowing the transfer function between the excitation antenna and the E field component at a point of interest. It is therefore sensible to define the gain in the average peak-field efficiency $G=\mathrm{E}\left[\eta_{\mathrm{CW}}\right] / \mathrm{E}\left[\eta_{\mathrm{TR}}\right]$. Applying CauchySchwarz inequality to (10) yields the following bound

$$
\mathrm{E}\left[\eta_{\mathrm{TR}}\right] \leq 2 \mathrm{E}\left[\int_{B_{T}}\left|\phi_{p}(f)\right|^{2} \mathrm{~d} f\right]=2 \int_{B_{T}} \phi_{\mathrm{av}}^{2}(f) \mathrm{d} f
$$

This results in an equality for a constant $P(f)$. The term under the integral sign is the same as in (7) and assuming it to be constant over the entire bandwidth of the pulse $p(t)$, we obtain

$$
G \leq \frac{B_{T} Q}{\pi f_{c}}
$$

This result is of paramount importance, since it allows assessing in a very simple way how time-reversal techniques would improve the performance of RC. Furthermore, it is a tool for designing the use of such techniques, as soon as the $Q$ of the $\mathrm{RC}$ is known. In the following experimental validation we will consider a constant $P(f)$, in order to meet the upper bound. 


\section{EXPERIMENTAL RESULTS}

The performance predicted by (12) was tested against experimental results measured in Supélec's RC $\left(3.08 \times 1.84 \times 2.44 \mathrm{~m}^{3}\right)$, using a log-periodic dipole antenna positioned near one corner of the chamber, with the dipoles of the antenna aligned along the vertical direction ( $z$ axis), while the direction of maximum gain was aimed at a corner. The electric field was sampled by means of an optical E-field probe, manufactured by Enprobe, model EFS-105. This probe is linearly polarized, so that three transfer functions were measured for the three Cartesian field components; to this effect, a styrofoam support was used. A grandtotal of 40 positions were considered, uniformly distributed over the lower half of the $\mathrm{RC}$, measuring the three Cartesian transfer functions by means of a network analyzer over three sub-bandwidths of $B_{T}=100 \mathrm{MHz}$, centered around central frequencies $f_{c}$ equal to $1,1.5$ and $2 \mathrm{GHz}$. Equations (6) and (10) were applied to each transfer function, obtaining the peak-field generation efficiencies; these results are presented in Fig. 1, where for each $f_{c}$, the average $\eta_{\mathrm{CW}}$ was set as reference, with all the results presented normalized to this value.

This procedure was then repeated with a reduced $B_{T}$, namely for $50 \mathrm{MHz}$ and $25 \mathrm{MHz}$ bandwidths, and the gain $G$ was computed. The results summarized in Table I prove that (12), despite its simplicity, allows assessing quite accurately the improvement in peak-field generation efficiency when adopting time-reversal techniques. The agreement is stronger at higher frequencies, where the field statistics in the RC are closer to an ideal reverberating medium [4]. Table I also demonstrates another interesting feature: time-reversal-driven RCs generate peak fields that are affected by a statistical dispersion by far lower than for a harmonic excitation. This feature is related to the self-averaging properties of time-reversal techniques, as already pointed out in [5] and [6].

\section{CONCLUSIONS}

We have proven that time-reversal techniques are an interesting alternative to the standard harmonic excitation of RCs, by showing that higher peak-field values can be generated from the same amount of energy. This feature was demonstrated experimentally and predicted by means of a simple asymptotic model. More reliable performances were also observed, with a strong reduction of the statistical dispersion of the peak-field amplitude.

\section{REFERENCES}

[1] H. Moussa, A. Cozza, and M. Cauterman, "Directive wavefronts inside a time reversal electromagnetic chamber," in IEEE EMC Symposium, Austin, Texas, 2009. 
[2] G. Lerosey, J. De Rosny, A. Tourin, A. Derode, G. Montaldo, and M. Fink, "Time reversal of electromagnetic waves," Physical Review Letters, vol. 92, no. 19, p. 193904, 2004.

[3] A. Cozza and H. Moussa, "Polarization selectivity for pulsed fields in a reverberation chamber," unpublished, currently submitted to Asia-Pacific EMC Symposium 2010, Beijing.

[4] D. Hill, "Plane wave integral representation for fields in reverberationchambers," IEEE Transactions on Electromagnetic Compatibility, vol. 40, no. 3, pp. 209-217, 1998.

[5] J. Fouque, J. Garnier, G. Papanicolaou, and K. Solna, Wave propagation and time reversal in randomly layered media. Springer, 2007.

[6] A. Cozza, "Statistics of the Performance of Time Reversal in a Lossy Reverberating Medium," Physical Review E, accepted for publication.

\section{AUTHOR'S AFFILIATION}

Andrea Cozza, e-mail : andrea.cozza@supelec.fr (Département de Recherche en Électromagnétisme, SUPELEC, 3 Rue Joliot-Curie, 91192 Gif sur Yvette). 


\section{FIGURE CAPTIONS}

Fig. 1 : Frequencies of occurrence distributions of $\eta_{\mathrm{CW}}$ (left column) and $\eta_{\mathrm{TR}}$ (right column), as obtained from transfer functions measured over a bandwidth $B_{T}=100 \mathrm{MHz}$, at a central frequency $f_{c}=\{1.0,1.5,2.0\} \mathrm{GHz}$ (top to bottom, respectively). All the results have been normalized to the average values of $\eta_{\mathrm{CW}}$.

Table 1 : Comparison of the efficiency gain $G$ assessed from experimental data and predicted by (12). Only the results for the field generated along the $x$-axis are shown for time-reversal excitation. The ratio of the normalized statistical dispersions is shown in the fifth column. 


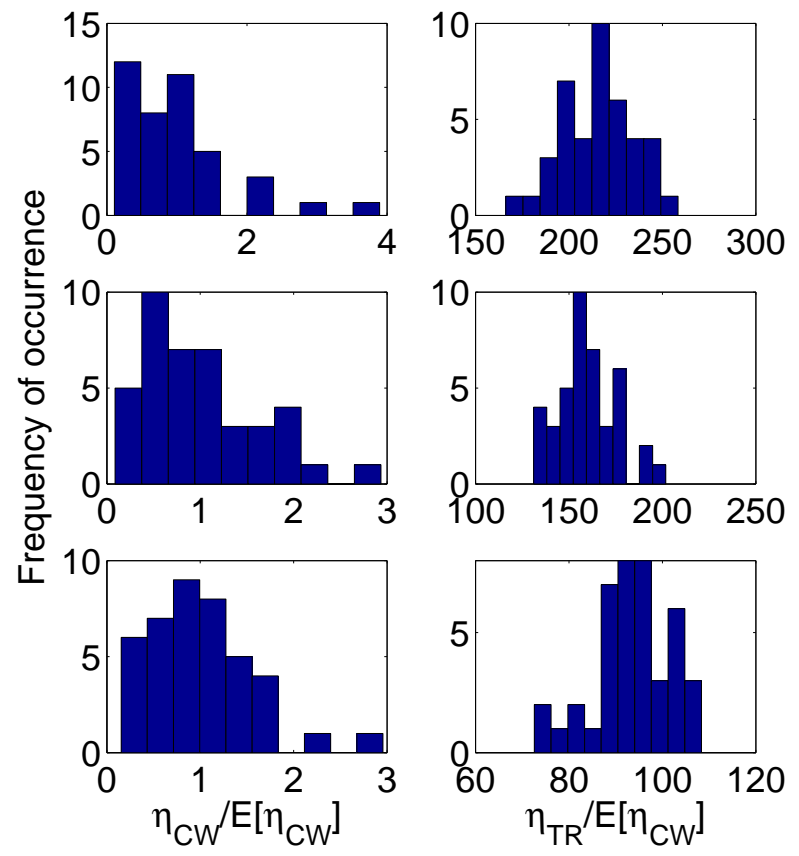

Fig. 1. 


\begin{tabular}{|cc|cccc|}
\hline $\begin{array}{c}f \\
(\mathrm{GHz})\end{array}$ & $\begin{array}{c}B_{T} \\
(\mathrm{MHz})\end{array}$ & $G$ & $\frac{B_{T} Q}{\pi f_{c}}$ & $\frac{(\sigma / \mu)_{\mathrm{CW}}}{(\sigma / \mu)_{\mathrm{TR}}}$ & $Q$ \\
\hline \multirow{2}{*}{1.0} & 25 & 60 & 45 & 7.7 & \\
& 50 & 116 & 91 & 9.3 & 5700 \\
& 100 & 218 & 182 & 11.9 & \\
\hline \multirow{2}{*}{1.5} & 25 & 39 & 34 & 6.3 & \\
& 50 & 80 & 68 & 7.0 & 6300 \\
& 100 & 161 & 135 & 7.7 & \\
\hline \multirow{2}{*}{2.0} & 25 & 24 & 25 & 6.5 & \\
& 50 & 47 & 50 & 7.6 & 6300 \\
& 100 & 95 & 100 & 8.5 & \\
\hline
\end{tabular}

TABLE I 